\begin{tabular}{|c|c|}
\hline Title & Estimation of salient regions related to chronic gastritis using gastric $X$-ray images \\
\hline Author(s) & Togo, Ren; Ishihara, Kenta; Ogawa, Takahiro; Hasey ama, Miki \\
\hline Citation & $\begin{array}{l}\text { Computers in biology and medicine, 77, 9-15 } \\
\text { https://doi.org/10.1016/.compbiomed.2016.07.014 }\end{array}$ \\
\hline Issue Date & 2016-10-01 \\
\hline Doc URL & http:/hdl .handle.net/2115/71556 \\
\hline Rights & $\begin{array}{l}\text { @ 2016. This manuscript version is made available under the CC-BY-NC-ND } 4.0 \text { license } \\
\text { http://creativecommons.org/icenses/by-nc-nd/4.0/ }\end{array}$ \\
\hline Rights(URL) & http://creativecommons.org/icenses/by-nc-nd/4.0/ \\
\hline Type & article (author version) \\
\hline File Information & revise_cbm_final_black-1.pdf \\
\hline
\end{tabular}

Instructions for use 


\title{
Estimation of Salient Regions Related to Chronic Gastritis Using Gastric $\mathrm{X}$-ray Images
}

\author{
Ren Togo, Kenta Ishihara, Takahiro Ogawa, Miki Haseyama \\ Graduate School of Information Science and Technology, Hokkaido University, Kita-14, Nishi-9, Sapporo-shi, 060-0814 Japan \\ TEL: +81-11-706-6078, FAX: +81-11-706-7369 \\ E-mail: \{togo,ishihara,ogawa\}@lmd.ist.hokudai.ac.jp, miki@ist.hokudai.ac.jp
}

\begin{abstract}
Since technical knowledge and a high degree of experience are necessary for diagnosis of chronic gastritis, computer-aided diagnosis (CAD) systems that analyze gastric X-ray images are desirable in the field of medicine. Therefore, a new method that estimates salient regions related to chronic gastritis/non-gastritis for supporting diagnosis is presented in this paper. In order to estimate salient regions related to chronic gastritis/non-gastritis, the proposed method monitors the distance between a target image feature and Support Vector Machine (SVM)-based hyperplane for its classification. Furthermore, our method realizes removal of the influence of regions outside the stomach by using positional relationships between the stomach and other organs. Consequently, since the proposed method successfully estimates salient regions of gastric $\mathrm{X}$-ray images for which chronic gastritis and non-gastritis are unknown, visual support for inexperienced clinicians becomes feasible.

Keywords: Chronic gastritis, helicobacter pylori, computer-aided diagnosis, radiology, support vector machine, region estimation.
\end{abstract}

\section{Introduction}

It has been revealed that Helicobacter pylori (H. pylori) infection causes the development of gastric cancer (Uemura et al., 2001). H. pylori is a bacterium that triggers the onset of gastritis, stomach ulcer, chronic atrophic gastritis. Although several etiologies like autoimmune, drug induced and infectious disease can cause chronic gastritis, the most common etiology is H. pylori-induced chronic gastritis. Therefore, we focus on $H$. pylori-induced chronic gastritis in this study and call these reactions as chronic gastritis. Chronic gastritis is considered to be the first step of mucosal changes in the stomach leading to gastric cancer, and diagnosis of it will allow the subsequent elimination of $H$. pylori and reduction in the risk of gastric cancer (Fukase et al., 2008; Fuccio et al., 2009). Therefore, screening for chronic gastritis has been used for identifying patients with the risk of gastric cancer.

Recently, it has been reported that chronic gastritis can be visually detected from gastric X-ray images in stomach fluoroscopy with high reliability by clinicians (Yamamichi et al., 2015). In stomach fluoroscopy, 
gastric X-ray images are examined carefully and thoroughly by focusing on folds and mucosal surfaces in the stomach. Although stomach fluoroscopy is one of the common screening examinations in Japan, there are two main problems. One problem is the large amount of workload. Clinicians have to read many gastric X-ray images per patient, and technical knowledge and a high degree of experience are required for accurate interpretation of the images. The other problem is the decreasing number of clinicians who have sufficient experience and technical knowledge for reading gastric X-ray images. In order to overcome the above problems, computer-aided diagnosis (CAD) systems that analyze gastric X-ray images are desirable, and such systems would enable rapid and precise diagnosis (Doi, 2005). Therefore, we previously proposed a method for chronic gastritis detection using gastric X-ray images to realize a CAD system (Ishihara et al., 2015). This method can automatically provide results of chronic gastritis classification with a high level of accuracy. However, even though the classification results for chronic gastritis is provided, inexperienced clinicians cannot recognize regions in which developing abnormalities exist in the stomach. Therefore, a system that not only provides results of classification but also supports a clinician's diagnosis visually is needed in the field of medicine (Lee \& Chen, 2015). A CAD system that can provide visual support, i.e., estimation of salient regions related to chronic gastritis/non-gastritis, would enable reduction in the workload of clinicians and rapid and precise diagnosis.

In the field of computer vision, many methods for computing salient regions within a target image have been proposed (Koch \& Ullman, 1987)-(Tian et al., 2014). Typical methods for estimating a meaningful region or object in an image depend on its rarity related to the edge, color, and boundaries. Unfortunately, it is difficult for such methods to estimate an area related to chronic gastritis/non-gastritis since visual characteristics of gastric X-ray images are unique. Therefore, a new method for realizing visual support for diagnosis of chronic gastritis is required.

In this paper, we present a new method to estimate salient regions related to chronic gastritis/nongastritis for realizing a visual support CAD system. The following two novel procedures are used in the proposed method: (i) calculation of contribution degrees for the classification of chronic gastritis/nongastritis and (ii) removal of the influence of regions outside the stomach. First, in (i), we monitor the distance between a visual feature vector calculated from a target image and a hyperplane calculated for the classification based on a Support Vector Machine (SVM) (Cortes \& Vapnik, 1995) to derive the contribution degree. Next, in (ii), we estimate regions of the stomach by focusing on the positional relationships between the stomach and other organs, and the influence of regions outside the stomach is removed by monitoring the contribution degrees derived in the above procedure. Finally, by combining the results obtained from the above two procedures, salient regions related to chronic gastritis/non-gastritis can be successfully estimated. Although our method is based on a simple scheme, it is the first work that realizes visual support for inexperienced clinicians. 
This paper is organized as follows. In Section 2, a brief explanation of the SVM used in the proposed method is given as preliminaries. The proposed method that estimates salient regions related to chronic gastritis/non-gastritis is presented in Section 3. Experimental results and discussion to verify the effectiveness of the proposed method are shown in Sections $\mathbf{4}$ and 5. Concluding remarks are given in Section 6.

\section{Support Vector Machine}

In this section, an SVM (Cortes \& Vapnik, 1995) for two-class classification is briefly explained as preliminaries. The goal of the SVM is to find an optimal separating hyperplane to maximize the margin, i.e., the distance between the hyperplane and the closest point of both classes. Given a training dataset that is composed of $L$ vectors $\boldsymbol{x}^{i} \in \mathbb{R}^{d}(i=1,2, \cdots, L)$ and their corresponding class labels $y^{i} \in\{1,-1\}$, the feature vectors $\boldsymbol{x}^{i}$ are mapped into a high-dimensional feature space to obtain $\Phi\left(\boldsymbol{x}^{i}\right) \in \mathbb{R}^{d^{\prime}}\left(d^{\prime} \gg d\right)$. Then the optimal hyperplane is defined as:

$$
f_{\mathrm{svm}}(\boldsymbol{x})=\boldsymbol{w}^{\top} \Phi(\boldsymbol{x})+b
$$

where $\boldsymbol{x}$ is an input vector whose class label is unknown, $\boldsymbol{w}$ is an adaptive weighted vector and $b$ is a bias. Furthermore, the class label is determined according to $y=\operatorname{sign}\left[f_{\mathrm{svm}}(\boldsymbol{x})\right]$.

The SVM calculates the separating hyperplane, i.e., $\boldsymbol{w}^{\top} \Phi(\boldsymbol{x})+b=0$, with the largest margin. The final result of Eq. (1) using a Lagrangian formulation can be rewritten as follows:

$$
f_{\mathrm{svm}}(\boldsymbol{x})=\sum_{i=1}^{L} \alpha^{i} y^{i} k\left(\boldsymbol{x}^{i}, \boldsymbol{x}\right)+b
$$

where $k(\cdot, \cdot)$ is a kernel function, and the coefficients $\alpha^{i}$ are obtained by solving the following quadratic programming problem:

$$
\begin{array}{lc}
\max & W(\alpha)=\sum_{i=1}^{L} \alpha^{i}-\frac{1}{2} \sum_{i, j=1}^{L} \alpha^{i} \alpha^{j} y^{i} y^{j} k\left(\boldsymbol{x}^{i}, \boldsymbol{x}\right), \\
\mathrm{s.t.} & \sum_{i=1}^{L} \alpha^{i} y^{i}=0, \\
& 0 \leq \alpha^{i} \leq C,(i=1,2, \cdots, L),
\end{array}
$$

where $\alpha^{i}$ correspond to the Lagrange multipliers, and $C$ is a parameter denoting the trade-off between the margin width and the training data error.

It should be noted that an output value of $f_{\mathrm{svm}}(\boldsymbol{x})$ is on the basis of the distance between $\Phi(\boldsymbol{x})$ and the 
hyperplane, and it can represent the degree of how the input feature vector is likely to be a positive/negative sample(Platt \& Others, 1999)-(Yamasaki \& Chen, 2012). Therefore, in this paper, we employ the output value of $f_{\mathrm{svm}}(\boldsymbol{x})$ for estimating salient regions related to chronic gastritis/non-gastritis.

\section{Salient Region Estimation}

A method for estimating salient regions related to chronic gastritis/non-gastritis is described in this section. First, since differences of chronic gastritis/non-gastritis can be observed in small regions, we divide the gastric X-ray images into blocks and extract image features for each block. Secondly, we employ a separating hyperplane of the SVM to derive the contribution degree for each block. Then salient regions related to chronic gastritis/non-gastritis can be estimated by two contribution maps based on a patient's individual condition and positional relationships of organs that are obtained from the derived contribution degrees.

In this section, extraction of image features is explained in 3.1, and a method for calculating the contribution degree for each block is shown in 3.2. The salient region estimation method is presented in 3.3.

\subsection{Feature Extraction}

In this subsection, we explain feature extraction from gastric X-ray images to recognize the differences of chronic gastritis/non-gastritis. As shown in Figure. 1, there are some differences between images with chronic gastritis and non-gastritis. Fig. 1 (a) shows an example of an chronic gastritis image (hereafter, a positive image), and Fig. 1 (b) shows an example of a non-gastritis image (hereafter, a negative image). In more detail, a stomach with chronic gastritis has coarse mucosal surface patterns and wide, non-straight and large shape folds. On the other hand, a stomach with non-gastritis has uniform and fine mucosal surface patterns and slim, straight and small shape folds.

To recognize these differences, we extract features shown in Table 1: texture features, shape features, and gradient-based features(Ishihara et al., 2015). In the proposed method, we divide each gastric X-ray image into $H \times W$ blocks ( $H$ and $W$ being the numbers of vertical blocks and horizontal blocks, respectively), and the above features are calculated for each block $b^{(h, w)}((h, w)=(1,1),(1,2), \cdots,(2,1), \cdots,(H, W) ;(h, w)$ being an axis of these blocks). In the proposed method, the calculated features are defined as a feature vector. Then the feature vector obtained from each gastric X-ray image is used in the calculation of the contribution degree shown in the following subsection.

\subsection{Calculation of Contribution Degree}

A method for calculating the contribution degree for each block $b^{(h, w)}$ is presented in this subsection. Let $F_{\text {train }}^{l}(l=1,2, \cdots, L)$ denote a gastric X-ray image, where $L$ is the number of training samples. Note 
that the class labels ( chronic gastritis/non-gastritis) of these $L$ images are known. Furthermore, $\boldsymbol{v}_{\text {train }}^{l,(h, w)} \in \mathbb{R}^{d}$ is defined as a feature vector calculated from a block $b^{(h, w)}$ of the $l$ th image, where $d=101$ as shown in Table 1. Then $\boldsymbol{x}_{\text {train }}^{l} \in \mathbb{R}^{D}(D=d \times(H \times W))$ is obtained by concatenating $\boldsymbol{v}_{\text {train }}^{l,(h, w)}$ for all of the blocks as follows:

$\boldsymbol{x}_{\text {train }}^{l}=\left[v_{\text {train }}^{l,(1,1)^{\top}}, v_{\text {train }}^{l,(1,2)^{\top}}, \cdots, v_{\text {train }}^{l,(h, w)^{\top}}, \cdots, v_{\text {train }}^{l,(H, W)^{\top}}\right]^{\top}$.

In the proposed method, we calculate a separating hyperplane of the SVM from $\boldsymbol{x}_{\text {train }}^{l}(l=1,2, \cdots, L)$ and their known class labels of chronic gastritis/non-gastritis. As mentioned in the previous section, the output value of $f_{\mathrm{svm}}(\cdot)$ is based on a distance from the hyperplane and it can be used as a confidence factor of classification(Platt \& Others, 1999)-(Yamasaki \& Chen, 2012).

Next, let $F_{\text {test }}$ denote a gastric X-ray image whose class label is unknown. Furthermore, $\boldsymbol{v}_{\text {test }}^{(h, w)} \in \mathbb{R}^{d}$ is a feature vector calculated from block $b^{(h, w)}$, and a feature vector $\boldsymbol{x}_{\text {test }} \in \mathbb{R}^{D}$ is defined as:

$\boldsymbol{x}_{\text {test }}=\left[\boldsymbol{v}_{\text {test }}^{(1,1)^{\top}}, \boldsymbol{v}_{\text {test }}^{(1,2)^{\top}}, \cdots, \boldsymbol{v}_{\text {test }}^{(h, w)}, \cdots, \boldsymbol{v}_{\text {test }}^{(H, W)^{\top}}\right]^{\top}$,

in the same manner as Eq. (4). First, we focus on the distance between the above feature vector and the generated hyperplane, and a standard confidence value is defined as follows:

$$
p_{\text {test }}=f_{\text {svm }}\left(\boldsymbol{x}_{\text {test }}\right)
$$

where $f_{\text {svm }}\left(\boldsymbol{x}_{\text {test }}\right)$ is the output value of the SVM obtained from $\boldsymbol{x}_{\text {test }}$, and it can be used as the confidence factor of the classification. Moreover, the result of classification can be determined by using $p_{\text {test }}$. If $p_{\text {test }} \geq 0$, the classification result becomes positive. Otherwise, the classification result becomes negative.

Next, in order to calculate the contribution degree of each block $b^{(h, w)}$ for the classification, we first define a mean vector $\overline{\boldsymbol{m}}_{\text {train }}^{(h, w)}\left(=\frac{1}{L} \sum_{l=1}^{L} \boldsymbol{v}_{\text {train }}^{l,(h, w)}\right)$ that is obtained from the feature vectors $\boldsymbol{v}_{\text {train }}^{l,(h, w)}$ belonging to block $b^{(h, w)}$. If the training images consist of almost the same numbers of chronic gastritis/non-gastritis images, $\overline{\boldsymbol{m}}_{\text {train }}^{(h, w)}$ contains both chronic gastritis and non-gastritis features. Thus, it can be assumed that $\overline{\boldsymbol{m}}_{\text {train }}^{(h, w)}$ becomes a feature vector that is difficult to be identified. Therefore, $\overline{\boldsymbol{m}}_{\text {train }}^{(h, w)}$ can be regarded as a feature vector that tends not to affect the classification.

As shown in Figure. 2, we replace $\boldsymbol{v}_{\text {test }}^{(h, w)}$ with $\overline{\boldsymbol{m}}_{\text {train }}^{(h, w)}$ to calculate the contribution of block $b^{(h, w)}$ for the classification. Specifically, the input vector after replacing $\boldsymbol{v}_{\text {test }}^{(h, w)}$ with $\overline{\boldsymbol{m}}_{\text {train }}^{(h, w)}$ is defined as:

$\boldsymbol{x}_{\text {test }}^{(h, w)}=\left[\boldsymbol{v}_{\text {test }}^{(1,1)^{\top}}, \boldsymbol{v}_{\text {test }}^{(1,2)^{\top}}, \cdots, \overline{\boldsymbol{m}}_{\text {train }}^{(h, w)^{\top}}, \cdots, \boldsymbol{v}_{\text {test }}^{(H, W)^{\top}}\right]^{\top}$. 
Then we recalculate the output value of the SVM as follows:

$$
p_{\text {test }}^{(h, w)}=f_{\mathrm{svm}}\left(\boldsymbol{x}_{\text {test }}^{(h, w)}\right)
$$

Since the distance from $p_{\text {test }}$ in Eq. (6) to $p_{\text {test }}^{(h, w)}$ in Eq. (8) is considered as the difference in the confidence of block $b^{(h, w)}$, we focus on this distance. Therefore, the contribution degree of block $b^{(h, w)}$ is defined as follows:

$C_{\text {test }}^{(h, w)}=\left\{\begin{array}{ll}\left|p_{\text {test }}\right|-\left|p_{\text {test }}^{(h, w)}\right| & \left(p_{\text {test }} \cdot p_{\text {test }}^{(h, w)} \geq 0\right) \\ \left|p_{\text {test }}\right|+\left|p_{\text {test }}^{(h, w)}\right| & \left(p_{\text {test }} \cdot p_{\text {test }}^{(h, w)}<0\right)\end{array}\right.$,

where $C_{\text {test }}^{(h, w)}$ represents the contribution for the classification in block $b^{(h, w)}$. Furthermore, $C_{\text {test }}^{(h, w)}$ considers the difference in the classification results of $p_{\text {test }}$ and $p_{\text {test }}^{(h, w)}$. Note that the contribution degree $C_{\text {test }}^{(h, w)}$ depends on the standard confidence value $p_{\text {test }}$, i.e., if the classification result is positive $\left(p_{\text {test }} \geq 0\right), C_{\text {test }}^{(h, w)}$ represents the contribution for chronic gastritis. On the other hand, if the classification result is negative $\left(p_{\text {test }}<\right.$ $0), C_{\text {test }}^{(h, w)}$ shows the contribution for non-gastritis. Therefore, in the proposed method, $C_{\text {test }}^{(h, w)}$ is used for estimating salient regions related to chronic gastritis/non-gastritis as shown in the following subsection.

\subsection{Estimation of Salient Regions}

In this subsection, we explain the method by which two patterns of contribution maps based on contribution degrees are derived and the obtained two contribution maps are integrated for estimating salient regions related to chronic gastritis/non-gastritis.

First, we use the contribution degree $C_{\text {test }}^{(h, w)}$ that is obtained from each test gastric X-ray image to estimate salient regions related to chronic gastritis/non-gastritis. The larger $C_{\text {test }}^{(h, w)}$ becomes, the larger becomes the contribution of block $b^{(h, w)}$ for the classification. Furthermore, $C_{\text {test }}^{(h, w)}$ depends on each patient's individual condition. Therefore, the contribution map based on each patient's individual condition can be directly defined as $M_{\text {Ind }}^{(h, w)}=C_{\text {test }}^{(h, w)}$.

Secondly, we derive another contribution map for removing the influence of regions outside the stomach. Since clinicians generally examine regions in the stomach to diagnose chronic gastritis/non-gastritis, regions outside the stomach are not required for the diagnosis. In the proposed method, we focus on the positional relationships between the stomach and other organs in a gastric X-ray image to remove such regions. Specifically, since the positional relationships between the stomach and other organs are invariable, it can be considered that gastric X-ray images in block $b^{(h, w)}$ are approximately located on the same organ. Therefore, we employ leave-one-out cross validation to calculate contribution degrees for all training images and use the mean of contribution degrees $\bar{C}_{\text {train }}^{(h, w)}\left(=\frac{1}{L} \sum_{l=1}^{L} C_{\text {train }}^{l,(h, w)}\right)$ in our method. Note that $C_{\text {train }}^{l,(h, w)}$ is 
the contributing degree of block $b^{(h, w)}$ in the $l$ th training image, which can be obtained in the same manner as $C_{\text {test }}^{(h, w)}$. Since $\bar{C}_{\text {train }}^{(h, w)}$ is obtained from $v_{\text {train }}^{l,(h, w)}(l=1,2, \cdots, L)$ belonging to block $b^{(h, w)}$, it can be considered that $\bar{C}_{\text {train }}^{(h, w)}$ represent a specific organ's contribution for the classification. Therefore, we define the contribution map based on this positional relationships for removing the influence of regions outside the stomach as $M_{\text {Pos }}^{(h, w)}=\bar{C}_{\text {train }}^{(h, w)}$.

Consequently, an integrated contribution map is derived by using the individual-based contribution map $M_{\mathrm{Ind}}^{(h, w)}$ and the positional relationships-based contribution map $M_{\mathrm{Pos}}^{(h, w)}$ as follows:

$$
S^{(h, w)}=M_{\mathrm{Ind}}^{(h, w)} \cdot M_{\mathrm{Pos}}^{(h, w)}
$$

Since the contribution map $S^{(h, w)}$ still contains blocks with noise, we remove such blocks by the opening processing (Serra, 1983) to obtain $S_{\text {Opening }}^{(h, w)}$. The final salient regions including saliency values can be obtained by:

$$
M_{\text {Final }}^{(h, w)}=\left\{\begin{array}{cl}
S_{\text {Opening }}^{(h, w)} & \text { if } S^{(h, w)}>\lambda \\
0 & \text { otherwise }
\end{array} .\right.
$$

Note that $\lambda$ is a threshold for deciding a salient block or non-salient block. The salient regions estimated by the proposed method consist of salient blocks with their saliency values. In this way, the estimation of salient regions related to chronic gastritis/non-gastritis become feasible by $M_{\text {Final }}^{(h, w)}$.

\section{Results}

An experiment was designed to evaluate the performance of the proposed method by using actual gastric X-ray images. Subsection 4.1 shows datasets and a verification method. Experimental results are presented in 4.2.

\subsection{Datasets and Verification Method}

In the experiment, we used a total of 1957 gastric X-ray images including 209 test images (97 positive images and 112 negative images) and 1748 training images (874 positive images and 874 negative images).Each image was 8-bit gray-scale and had a resolution of $1024 \times 1024$ pixels, and we set $H \times W$ to $16 \times 16$.

Since the most common etiology of chronic gastritis is H. pylori infection (Sipponen \& Maaroos, 2015), ABC classification(Kudo et al., 2011), which is a simple blood examination for $H$. pylori infection diagnosis based on serum H. pylori antibody levels (Kawai et al., 2002) and serum pepsinogen levels, was used as a ground truth of the training images. It should be noted that serum H. pylori antibody levels are used for 
estimation of $H$. pylori infection and serum pepsinogen levels are used to assess the degree of gastritis ${ }^{1}$. In more detail, $\mathrm{ABC}$ classification categories the gastric cancer risk into four groups, i.e., groups $\mathrm{A}, \mathrm{B}$, $\mathrm{C}$ and D. Concerning H. pylori infection, group A is negative (uninfected), and groups $\mathrm{B}, \mathrm{C}$ and $\mathrm{D}$ are positive (infected). Therefore, we defined samples in group A was non-gastritis and samples in groups B, C and D were chronic gastritis in this study. Concerning 209 test images, the number of cases in groups $\mathrm{A}, \mathrm{B}, \mathrm{C}$ and $\mathrm{D}$ from $\mathrm{ABC}$ classification was 112, 50, 40 and 7, respectively. Also, among 1748 training images, the number of cases in groups A, B, C and D from ABC classification was 874, 458, 380 and 36, respectively. Since ABC classification often has false negative samples, we excluded $H$. pylori eradicated samples and suspected false negative samples that are determined from group A base on values of $\mathrm{ABC}$ classification(PG I levels $\leq 30 \mathrm{ng} / \mathrm{ml}$, PG II levels $\geq 15 \mathrm{ng} / \mathrm{ml}$ or PG I / PG II $<4$ ).

The largest value of contribution degrees among outermost blocks of the contribution map $S^{(h, w)}$ was set as the threshold $(=\lambda)$ for deciding salient blocks or non-salient blocks. In order to confirm the effectiveness of salient regions estimated by the proposed method, SVM-based classification was applied to the following two kinds of feature vectors: (i) feature vectors calculated from only salient blocks and (ii) feature vectors calculated from all blocks. We utilized accuracy (Acc), sensitivity (Sen) and specificity (Spe), which are commonly used to evaluate reliability of clinical examinations in the field of medicine. We also utilized the Youden Index (YI) since sensitivity and specificity have trade-off relationship.

\subsection{Experimental Results}

In this subsection, we show our experimental results of salient regions calculated by the proposed method. Examples of gastric X-ray images used in this experiment are shown in Figure 3. Note that ABC classification results of three sample images shown in Fig. 3 (a)-(d) were A, B, C and D, respectively. First, Figures 4-6 show contribution maps $M_{\text {Ind }}^{(h, w)}, M_{\text {Final }}^{(h, w)}$ and $M_{\text {Pos }}^{(h, w)}$, respectively. In terms of contribution maps, the closer to red color the block becomes, the stronger is the relation for chronic gastritis/non-gastritis, whereas blue blocks are non-related for chronic gastritis/non-gastritis. As shown in Figs. 4 and 6, it can be seen that contribution degrees are different for each block and each gastric X-ray image.

Next, we compared the salient regions calculated by the proposed method and those calculated by conventional estimation methods. To the best of our knowledge, there have not been any proposed methods that estimate salient regions related to chronic gastritis/non-gastritis. Therefore, we performed the comparison with saliency estimation methods that are frequently used in the field of computer vision. Figures 7-9 show the salient regions estimated by our method, comparative method 1 (Graph-Based Visual Saliency model(Harel et al., 2006)) and comparative method 2 (Signature Saliency algorithm (Hou et al., 2012)),

\footnotetext{
${ }^{1}$ In this experiment, E Plate Eiken H. pylori Antibody (Eiken Chemical Co., Ltd., Tokyo, Japan) and Auto pepsinogen I BML-2G and Auto pepsinogen II BML-2(BML, Inc., Ltd., Saitama, Japan) were used for the ABC classification.
} 
respectively. From this experimental results, although salient regions obtained from the two comparative methods in Figs. 8 and 9 do not include regions of the stomach, salient regions obtained by our method in Fig. 7 are located in the stomach.

Moreover, we verified the effectiveness of salient regions obtained by the proposed method, quantitatively. If the salient regions estimated by our method have a relation to chronic gastritis/non-gastritis, successful classification of the SVM can be guaranteed by using visual features obtained from these regions. Table 2 provides the results of classification performance. As shown in Table 2, the use of salient blocks obtained by our method (method (i)) outperforms the comparative method (method (ii)) in accuracy. Although our method (method (i)) is inferior to method (ii) in specificity, there is a trade-off relationship between sensitivity and specificity as mentioned above. It can be seen in Table 2 that the performance of Youden Index considering both of sensitivity and specificity in method (i) is better than that of (ii). Specifically, in 209 test images, 162 cases were correctly classified in our method, the false negative was 18 cases, and the false positive was 21 cases.

\section{Discussion}

The focus of our study is to estimate salient regions related to chronic gastritis/non-gastritis using gastric $\mathrm{X}$-ray images for realizing a visual diagnosis supporting system. The obtained experimental results revealed that the proposed method can estimate subtle differences of chronic gastritis/non-gastritis as salient regions. Furthermore, we verified the effectiveness of the proposed method both subjectively and quantitatively. Therefore, successful estimation of salient regions related to chronic gastritis/non-gastritis can be achieved by the proposed method. Next, in the present study, many CAD systems based on a supervised classifier have been proposed, which provide classification results with a highly reliable level of accuracy (Miranda \& Felipe, 2015), (Balodi et al., 2016). In contrast to such CAD systems, the proposed method can be regarded as a boosting method for achieving successful classification to realize more practical CAD systems. Thus, the advantages of the proposed method are (i) it visualizes regions related to chronic gastritis to move toward an objective interpretation and (ii) classification performance improvement.

Concerning the results of classification performance in Table 2, the threshold determining chronic gastritis/non-gastritis can be continuously changed corresponding to demands of a clinician in our method. Next, in this experiment, we employed $\mathrm{ABC}$ classification results as a ground truth of training data. However, the gold standard to identify cases of $H$. pylori infection is biopsy based on endoscopy. Employing the training data proven in biopsy will refine our method. 


\section{Conclusions}

A method to estimate salient regions related to chronic gastritis/non-gastritis using gastric X-ray images is presented in this paper. The proposed method consists of two main procedures, calculation of contribution degrees and removal of regions outside the stomach. The effectiveness of the proposed method was evaluated subjectively and quantitatively by performing experiments using actual gastric X-ray images. Since the proposed method can be applied to gastric X-ray images for which chronic gastritis and non-gastritis are unknown, visual support for inexperienced clinicians becomes feasible.

\section{Conflicts of interest statement}

None declared.

\section{Acknowledgment}

In this research, we utilized the image data and the blood data kindly provided by the Medical Examination Center of the Yamagata City Medical Association. We would also like to sincerely thank to Katsuhiro Mabe of the Hakodate National Hospital, Shigemi Nakajima of the Social Insurance Shiga hospital, Harufumi Oizumi of the Yamagata City Medical Association, and Kazuya Yoshizawa of the Faculty of Medicine, Yamagata University, and many others, for providing data, images and results as well as invaluable advice. This research was partly supported by Griant-in Aid for Scientific Research (B) 25280036 from JSPS.

Balodi, A., Dewal, M. L., Anand, R. S., \& Rawat, A. (2016). Texture based classification of the severity of mitral regurgitation. Computers in Biology and Medicine, 73, 157-164.

Cortes, C., \& Vapnik, V. (1995). Support-vector networks. Machine learning, 20, 273-297.

Doi, K. (2005). Current status and future potential of computer-aided diagnosis in medical imaging. The British Journal of Radiology, $78, \mathrm{~s} 3-\mathrm{s} 19$.

Duda, R. O., \& Hart, P. E. (1972). Use of the Hough transformation to detect lines and curves in pictures. Communications of the ACM $15,11-15$.

Fuccio, L., Zagari, R. M., Eusebi, L. H., Laterza, L., Cennamo, V., Ceroni, L., Grilli, D., \& Bazzoli, F. (2009). Meta-analysis: Can Helicobacter pylori Eradication Treatment Reduce the Risk for Gastric Cancer? Annals of Internal Medicine, 151, $121-128$.

Fukase, K., Kato, M., Kikuchi, S., Inoue, K., Uemura, N., Okamoto, S., Terao, S., Amagai, K., Hayashi, S., Asaka, M., \& Others (2008). Effect of eradication of Helicobacter pylori on incidence of metachronous gastric carcinoma after endoscopic resection of early gastric cancer: an open-label, randomised controlled trial. The Lancet, 372, 392-397.

Gao, W., Zhang, X., Yang, L., \& Liu, H. (2010). An improved Sobel edge detection. In Computer Science and Information Technology (ICCSIT), 2010 3rd IEEE International Conference on (pp. 67-71). IEEE volume 5.

Haralick, R. M., Shanmugam, K., \& Dinstein, I. H. (1973). Textural features for image classification. Systems, Man and Cybernetics, IEEE Transactions on, (pp. 610-621).

Harel, J., Koch, C., \& Perona, P. (2006). Graph-based visual saliency. In Advances in neural information processing systems (pp. $545-552)$

Hou, X., Harel, J., \& Koch, C. (2012). Image Signature: Highlighting Sparse Salient Regions. Pattern Analysis and Machine Intelligence, IEEE Transactions on, 34, 194-201. 
Hu, M.-K. (1962). Visual pattern recognition by moment invariants. Information Theory, IRE Transactions on, 8, $179-187$.

Ishihara, K., Ogawa, T., \& Haseyama, M. (2015). Helicobacter pylori infection detection from multiple x-ray images based on combination use of support vector machine and multiple kernel learning. In Image Processing (ICIP), 2015 IEEE International Conference on (pp. 4728-4732).

Kawai, T., Kawakami, K., Kudo, T., Ogiahara, S., Handa, Y., \& Moriyasu, F. (2002). A new serum antibody test kit (E plate) forevaluation of helicobacter pylori eradication. Internal Medicine, 41, 780-783.

Koch, C., \& Ullman, S. (1987). Shifts in selective visual attention: towards the underlying neural circuitry. In Matters of intelligence (pp. 115-141). Springer.

Kudo, T., Kakizaki, S., Sohara, N., Onozato, Y., Okamura, S., Inui, Y., \& Mori, M. (2011). Analysis of ABC (D) stratification for screening patients with gastric cancer. World journal of gastroenterology: WJG, 17, 4793.

Lee, H., \& Chen, Y.-P. P. (2015). Image based computer aided diagnosis system for cancer detection. Expert Systems with Applications, $42,5356-5365$.

Lin, C.-H., Liu, C.-W., \& Chen, H.-Y. (2012). Image retrieval and classification using adaptive local binary patterns based on texture features. IET image processing, 6, 822-830.

Miranda, G. H. B., \& Felipe, J. C. (2015). Computer-aided diagnosis system based on fuzzy logic for breast cancer categorization. Computers in Biology and Medicine, 64, 334-346.

Platt, J., \& Others (1999). Probabilistic outputs for support vector machines and comparisons to regularized likelihood methods. Advances in large margin classifiers, 10, 61-74.

Serra, J. (1983). Image analysis and mathematical morphology. Academic Press, Inc.

Sipponen, P., \& Maaroos, H.-I. (2015). Chronic gastritis. Scandinavian journal of gastroenterology, 50, 657-67.

Tian, H., Fang, Y., Zhao, Y., Lin, W., Ni, R., \& Zhu, Z. (2014). Salient Region Detection by Fusing Bottom-Up and Top-Down Features Extracted From a Single Image. Image Processing, IEEE Transactions on, 23, 4389-4398.

Uemura, N., Okamoto, S., Yamamoto, S., Matsumura, N., Yamaguchi, S., Yamakido, M., Taniyama, K., Sasaki, N., \& Schlemper, R. J. (2001). Helicobacter pylori Infection and the Development of Gastric Cancer. New England Journal of Medicine, 345, 784-789.

Yamamichi, N., Hirano, C., Ichinose, M., Takahashi, Y., Minatsuki, C., Matsuda, R., Nakayama, C., Shimamoto, T., Kodashima, S., Ono, S., Tsuji, Y., Niimi, K., Sakaguchi, Y., Kataoka, Y., Saito, I., Asada-Hirayama, I., Takeuchi, C., Yakabi, S., Kaikimoto, H., Matsumoto, Y., Yamaguchi, D., Kageyama-Yahara, N., Fujishiro, M., Wada, R., Mitsushima, T., \& Koike, K. (2015). Atrophic gastritis and enlarged gastric folds diagnosed by double-contrast upper gastrointestinal barium X-ray radiography are useful to predict future gastric cancer development based on the 3-year prospective observation. Gastric Cancer, (pp. 1-7).

Yamasaki, T., \& Chen, T. (2012). Confidence-assisted classification result refinement for object recognition featuring TopN-ExemplarSVM. In Pattern Recognition (ICPR), 2012 21st International Conference on (pp. 1783-1786).

Table 1: Features used in the proposed method.

\begin{tabular}{llc}
\hline Feature Types & Details of Features & Dimensions \\
\hline \hline \multirow{2}{*}{ Texture features } & Gabor Wavelet-based features & $56 \times H \times W$ \\
& Adaptive Local Binary Pattern(Lin et al., 2012) & $22 \times H \times W$ \\
& Intensity histogram-related features & $4 \times H \times W$ \\
& Co-occurrence matrix-related features(Haralick et al., 1973) & $9 \times H \times W$ \\
\hline \multirow{2}{*}{ Shape features } & Hu-moment invariant(Hu, 1962) & $7 \times H \times W$ \\
& Hough transform-based features(Duda \& Hart, 1972) & $2 \times H \times W$ \\
\hline Gradient-based features & Sobel filter-based edge features(Gao et al., 2010) & $1 \times H \times W$ \\
\hline \hline
\end{tabular}




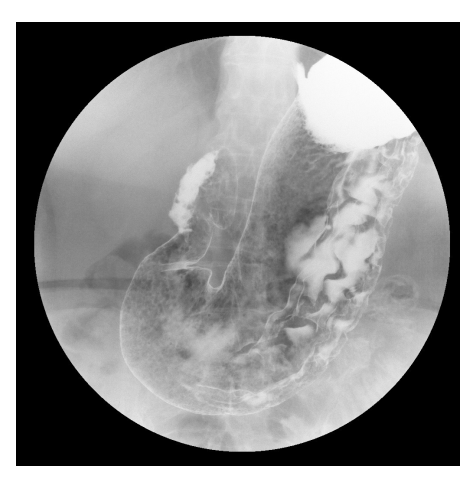

(a)

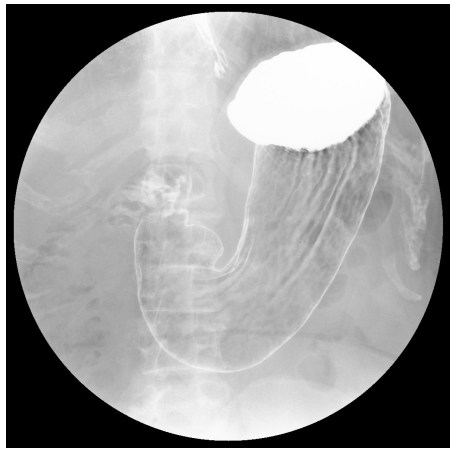

(b)

Figure 1: Examples of gastric X-ray images used in this study: (a) an example image of chronic gastritis and (b) an example image of non-gastritis.

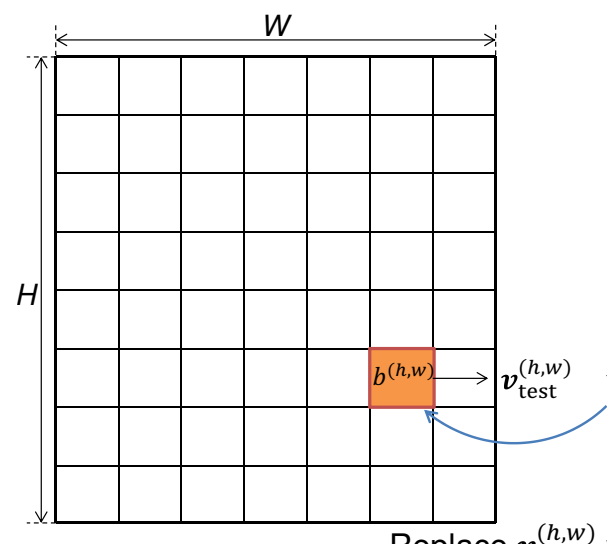

Test image $F_{\text {test }}$

Replace $\boldsymbol{v}_{\text {test }}^{(h, w)}$ with $\overline{\boldsymbol{m}}_{\text {trai }}^{(h, w)}$

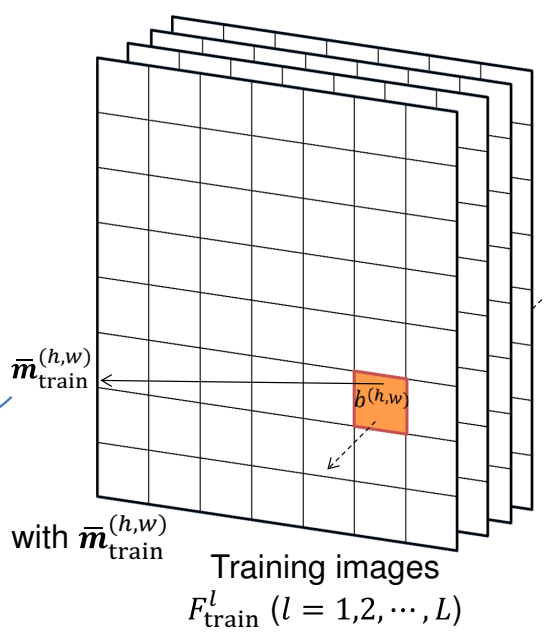

SVM classification

Figure 2: Procedures for replacing the feature vector $\boldsymbol{v}_{\text {test }}^{(h, w)}$ with $\overline{\boldsymbol{m}}_{\text {train }}^{(h, w)}$ for calculation of the contribution degree of block $b^{(h, w)}$.

Table 2: Results for Accuracy, Sensitivity, Specificity, and Youden index.

\begin{tabular}{lcccc}
\hline Methods & Acc & Sen & Spe & YI \\
\hline \hline (i) Using salient blocks & $\mathbf{0 . 7 7 5}$ & $\mathbf{0 . 8 1 4}$ & 0.741 & $\mathbf{0 . 5 5 5}$ \\
\hline (ii) Using all blocks & 0.732 & 0.680 & $\mathbf{0 . 7 7 7}$ & 0.457 \\
\hline
\end{tabular}




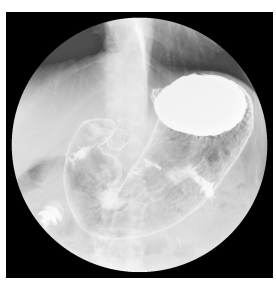

(a)

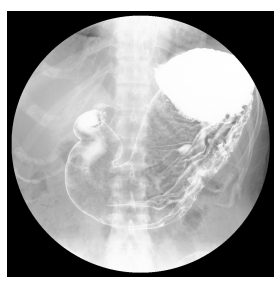

(b)

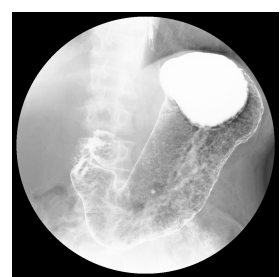

(c)

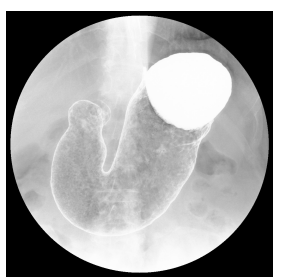

(d)

Figure 3: Examples of original gastric X-ray images: (a) is the negative image (the result of ABC classification was A) and (b), (c) and (d) are positive images (the results of $\mathrm{ABC}$ classification were $\mathrm{B}, \mathrm{C}$ and $\mathrm{D}$, respectively).

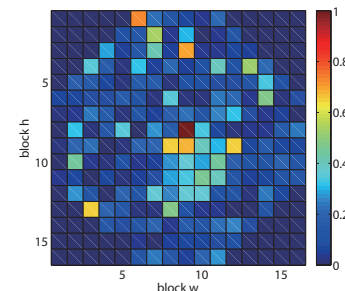

(a)

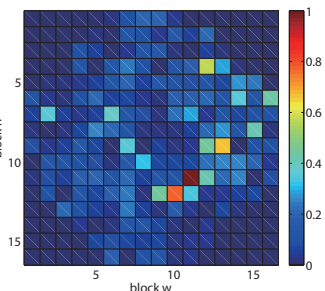

(b)

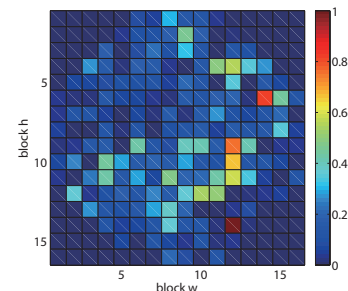

(c)

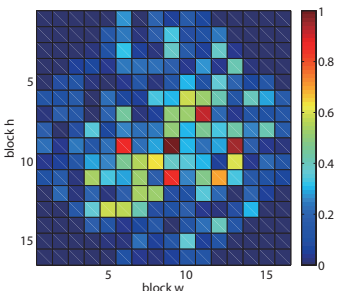

(d)

Figure 4: Contribution maps $M_{\text {Ind }}^{(h, w)}$ based on each patient's individual condition: (a) - (d) were obtained from Fig. 3 (a) - (d), respectively.

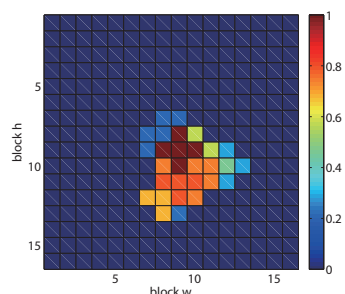

(a)

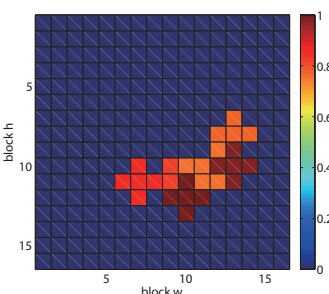

(b)

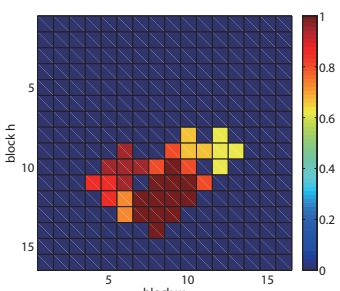

(c)

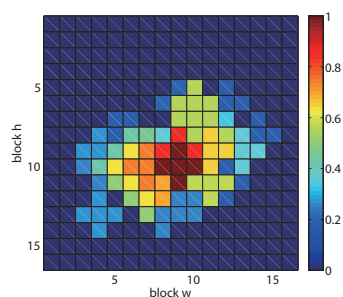

(d)

Figure 5: Final contribution maps $M_{\text {Final }}^{(h, w)}$ obtained by the proposed method: (a), (b), (c) and (d) were calculated from Figs. 4 (a), (b), (c), (d) and Fig. 6, respectively.

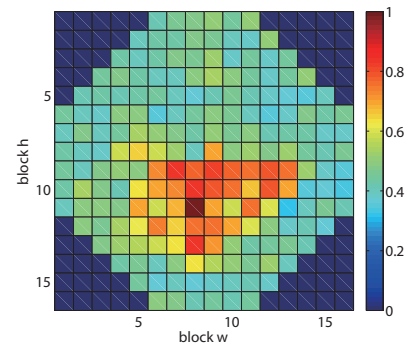

Figure 6: Contribution map $M_{\mathrm{Pos}}^{(h, w)}$ based on positional relationships: $M_{\mathrm{Pos}}^{(h, w)}$ was obtained by using all training images. 


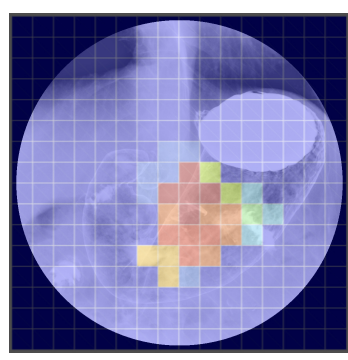

(a)

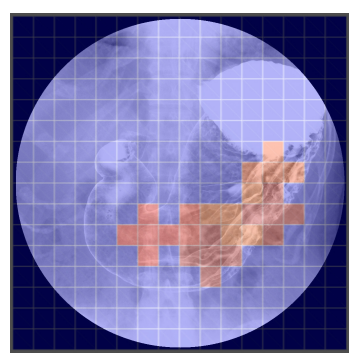

(b)

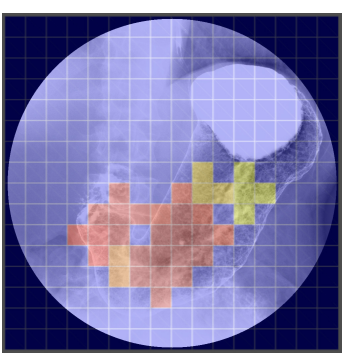

(c)

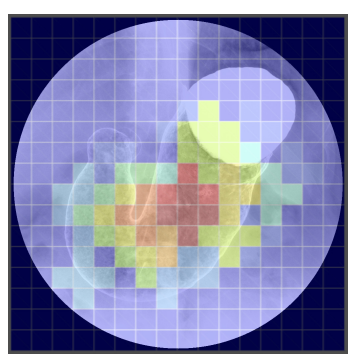

(d)

Figure 7: Salient regions obtained by the proposed method: (a) - (d) were obtained by superposing original gastric X-ray images in Fig. 3 and $M_{\text {Final }}^{(h, w)}$ in Fig. 5, respectively.

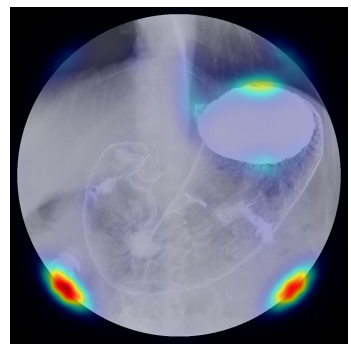

(a)

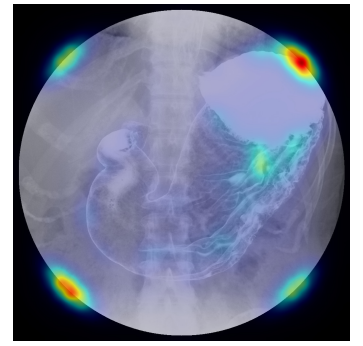

(b)

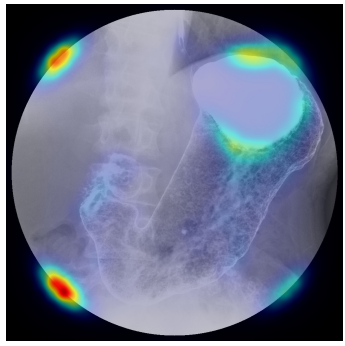

(c)

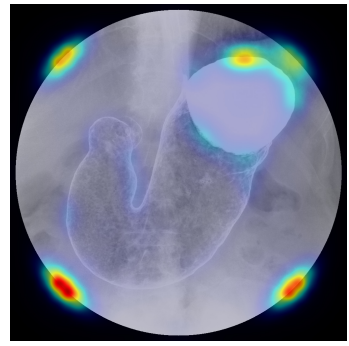

(d)

Figure 8: Results obtained by comparative method 1 that has been presented in (Harel et al., 2006).

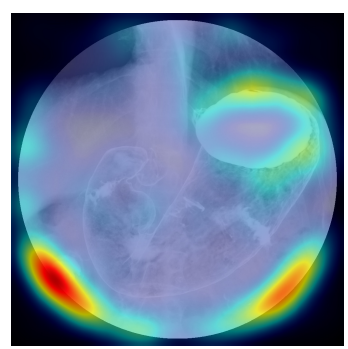

(a)

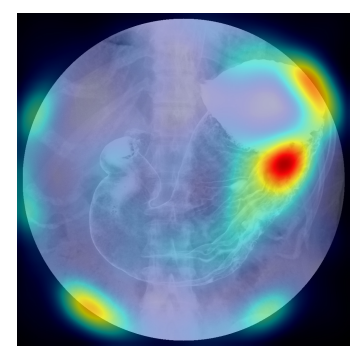

(b)

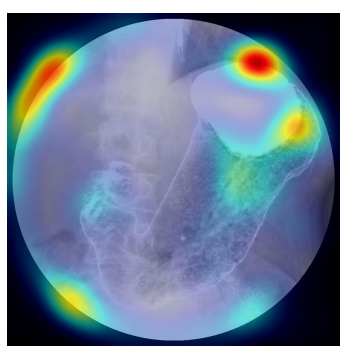

(c)

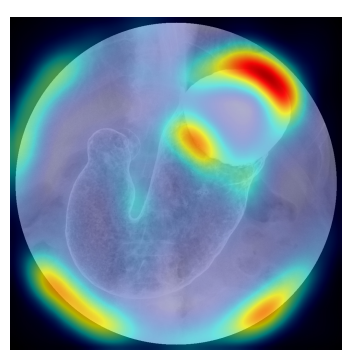

(d)

Figure 9: Results obtained by comparative method 2 that has been presented in (Hou et al., 2012). 